\title{
Article
}

\section{An Elementary Approximation of Dwell Time Algorithm for Ultra-Precision Computer-Controlled Optical Surfacing}

\author{
Yajun Wang ${ }^{1,2}$, Yunfei Zhang ${ }^{2, *}$, Renke Kang ${ }^{1}$ and Fang $\mathrm{Ji}^{2}$ \\ 1 School of Mechanical Engineering, Dalian University of Technology, Dalian 116023, China; \\ jjswang@xjtu.edu.cn (Y.W.); kangrk@dlut.edu.cn (R.K.) \\ 2 Institute of Machinery Manufacturing Technology, China Academy of Engineering Physics, \\ Mianyang 621900, China; jfang2013@caep.cn \\ * Correspondence: zhangyf306@yeah.net; Tel.: +86-0286-5726-617
}

check for updates

Citation: Wang, Y.; Zhang, Y.; Kang,

R.; Ji, F. An Elementary

Approximation of Dwell Time

Algorithm for Ultra-Precision

Computer-Controlled Optical

Surfacing. Micromachines 2021, 12, 471.

https://doi.org/10.3390/mi12050471

Academic Editor: Xichun Luo

Received: 23 March 2021

Accepted: 16 April 2021

Published: 21 April 2021

Publisher's Note: MDPI stays neutral with regard to jurisdictional claims in published maps and institutional affiliations.

Copyright: (c) 2021 by the authors. Licensee MDPI, Basel, Switzerland. This article is an open access article distributed under the terms and conditions of the Creative Commons Attribution (CC BY) license (https:// creativecommons.org/licenses/by/ $4.0 /)$.

\begin{abstract}
The dwell time algorithm is one of the key technologies that determines the accuracy of a workpiece in the field of ultra-precision computer-controlled optical surfacing. Existing algorithms mainly consider meticulous mathematics theory and high convergence rates, making the computation process more uneven, and the flatness cannot be further improved. In this paper, a reasonable elementary approximation algorithm of dwell time is proposed on the basis of the theoretical requirement of a removal function in the subaperture polishing and single-peak rotational symmetry character of its practical distribution. Then, the algorithm is well discussed with theoretical analysis and numerical simulation in cases of one-dimension and two-dimensions. In contrast to conventional dwell time algorithms, this proposed algorithm transforms superposition and coupling features of the deconvolution problem into an elementary approximation issue of function value. Compared with the conventional methods, it has obvious advantages for improving calculation efficiency and flatness, and is of great significance for the efficient computation of large-aperture optical polishing. The flatness of $\varphi 150 \mathrm{~mm}$ and $\varphi 100 \mathrm{~mm}$ workpieces have achieved $\mathrm{PVr}_{150}=0.028 \lambda$ and $\mathrm{PVCr}_{100}=0.014 \lambda$ respectively.
\end{abstract}

Keywords: ultra-precision machining; computer-controlled optical surfacing; dwell time algorithm; removal function; elementary approximation

\section{Introduction}

With the rapid increasing requirements for the fabrication of high-precision optical elements in modern optical systems, several advanced deterministic optical surfacing technologies have been developed over the past decades, such as ultra-precision computer controlled optical surfacing (CCOS), magnetorheological finishing (MRF), ion-beam figuring (IBF), bonnet polishing (BP) [1,2]. These achieve precision material removal on certain workpiece areas by accurately controlling the dwell time on the elaborately predesigned polishing path. Therefore, the dwell time algorithm is one of the key elements in modern advanced deterministic optical surfacing technologies. Among them, most of the removal functions have the characteristics of rotational symmetry, but how to use this feature to develop a high-efficiency, high-precision dwell time algorithm is the current research focus.

In deterministic optical finishing technologies, the amount of material removal can be expressed as discrete two-dimensional convolution operations of the dwell time and removal functions. The dwell time algorithm is used to solve the deconvolution process and to ensure that the calculated dwell time not only meets the performance of machine tools, but also has a high surface error convergence efficiency. Various dwell time algorithms have earlier been proposed, including the Fourier transform method, the numerical iteration method, the matrix equation method. Ronald Aspden et al. [3] studied the polar and rectangular coordinates of the process in CCOS, and discussed the variation of the removal function with the radius of the workpiece in the gyrosymmetric correction process. 
Jones et al. [4] proposed an iterative method for solving the dwell time function, studied the relationship between the flatness convergence efficiency and the removal function, and pointed out that only the symmetric central single-peak removal function could converge. The convergence accuracy of these methods is not high enough, and now they are seldom applied. Carnal et al. [5] introduced the linear equation method and solved the dwell time by adopting the LSQR method. Drueding et al. [6] proposed a series expansion solution. Waluschka et al. [7] presented a one-dimensional dwell time function algorithm for cylindrical workpieces based on a graphic method, and Shanbhag et al. [8] proposed an algorithm based on wavelet analysis. Zheng et al. [9] proposed a damped iterative method for solving the CCOS dwell time function. Zhou et al. [10] used the TSVD method to solve the linear equation model, which entailed further research. Wu et al. [11] proposed a solution based on discretized matrix equations using LSQR. Jiao et al. [12] and Jiang et al. [13] improved the traditional $\mathrm{L}-\mathrm{R}$ algorithm. Taking into account the scanning path of the polishing tool and the tilt angle of the workpiece, Guo [14] proposed a dwell time algorithm to achieve rapid convergence of the accuracy of the optical mold. Pan et al. [15] proposed an improved dwell time calculation algorithm to optimize tool path planning in optical figuring. Li et al. [16] developed a positive dwell time algorithm with minimum equal extra material removal to consider the machine dynamics limitations. Li and Zhou [17] gave a solution algorithm of dwell time in a slope-based figuring model. Wang et al. [18] provided a quantitative study on the performances of dwell time algorithms in ion-beam figuring. Han et al. $[19,20]$ proposed a Gaussian mixture model to model experimental removal functions and provided the dwell time algorithm according to the dynamic characteristics of the machine tool. These methods are mainly based on matrix equations, and the computational efficiency might be much lower especially for large-aperture optical elements, so the solution might not be smooth enough.

The existing dwell time algorithms are conducted mainly based on a meticulous mathematical theory and designed to pursue high convergence rate. Nonetheless, those methods do not adequately consider the distribution characteristics of the removal function and rarely incorporate the speed-smoothing issues that are closely related to convergence efficiency and machine tool motion implementation. Actually, the convergence rate of flatness is only between 1.1X and 1.3X [21] for most CNC machines; hence, it is unnecessary to pursue high convergence rates excessively.

In this paper, an elementary approximation method for solving the dwell time algorithm based on the symmetrical distribution of single-peak rotation of removal function is proposed. The proposed method has the characteristics of clear physical meaning and was verified by simulation and experiments. By using triangular approximation of the removal function, the initial surface shape is discretized reasonably and the approximate solution of dwell time is obtained. In this paper, the performance of the algorithm is verified through residence-time mathematical modeling, accuracy analysis, simulation and experimental research. The results showed that it performs well in the profile for smoothness and convergence efficiency.

\section{Dwell Time Algorithm Model}

\subsection{Approximation Treatment of Removal Function}

According to the measured residual error, the surface is polished to achieve a theoretical profile. To eliminate the residual error, the material removal function, generated by the polishing tool in a constant time (also called the removal function) [22-24], and the dwell time of the polishing tool should be known first. It is generally assumed that deterministic optical surfacing technology is a linear shift-invariant system, and the mathematical model of the convolution of the dwell time and removal functions being equal to the distribution of the removal amount is generally adopted, and this is given in Equation (1) [6]:

$$
\Delta h(x, y)=R(x, y) * * D(x, y)
$$


where $\Delta h(x, y)$ is the distribution function of removal amount, $R(x, y)$ is the removal function (also called influence function), and $D(x, y)$ is the dwell time function.

In some deterministic optical surfacing technologies, the removal function is distributed by rotational symmetry. Suppose an ideal removal function is a two-dimensional Gaussian distribution, as shown in Figure 1.

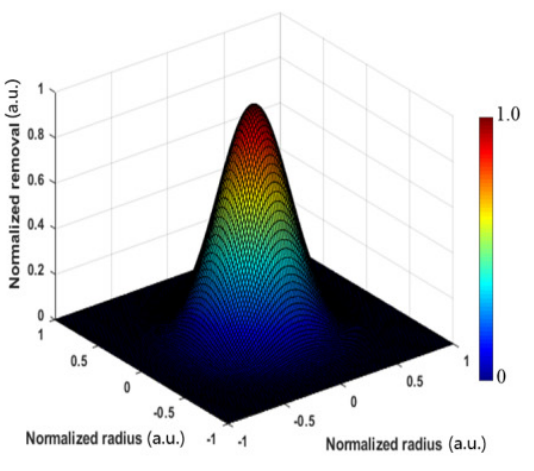

(a)

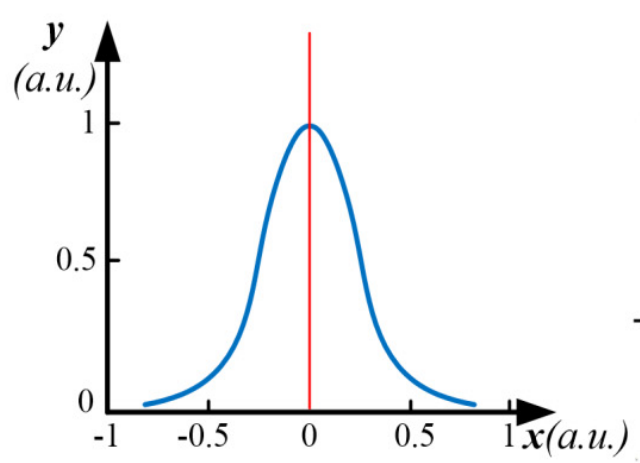

(b)

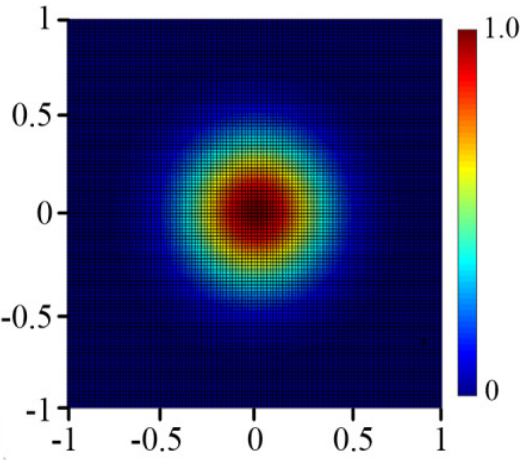

(c)

Figure 1. The basic distribution of removal function in some deterministic optical surfacing technologies: (a) Twodimensional distribution of ideal Gaussian removal function, (b) One-dimensional distribution of CCOS removal function, (c) Kernel function distribution of ion beam figuring (IBF).

According to the actual characteristics of its distribution [25], the removal function is approximated as shown in Figure 2.

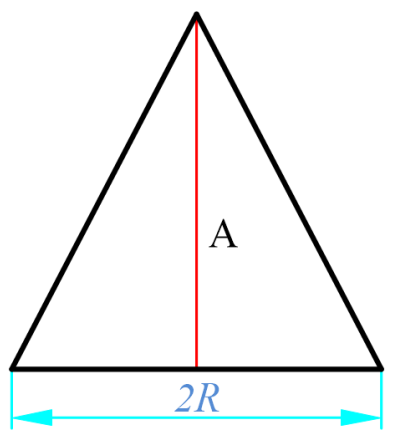

(a)

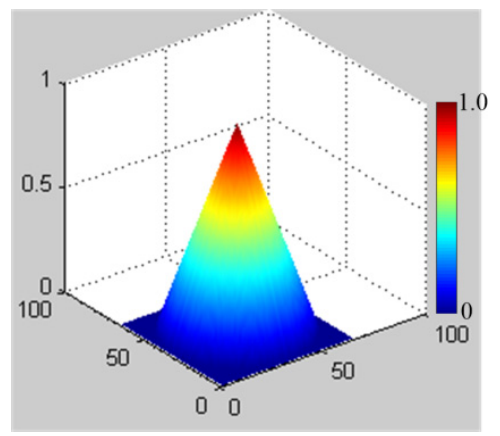

(b)

Figure 2. Approximate distribution of the removal function: (a) One-dimensional approximation, (b) Two-dimensional approximation.

In the one-dimensional case, the removal function can be represented by an isosceles triangle distribution with height $A$ and bottom $2 R$, similar to the roof function in the one-dimensional finite element method. In the two-dimensional case, it is assumed that the kernel function can be represented by a conic distribution with a height of $A$ and a bottom radius of $R$. This kind of approximation reflects the main distribution characteristics of the removal function and concentrates more than $80 \%$ of volume removal, which is completely acceptable in engineering.

\subsection{Dwell Time Algorithm Model}

\subsubsection{One-Dimensional Analysis}

The principle of an elementary approximation for a one-dimensional deconvolution is shown in Figure 3. The blue curve represents the target removal amount distribution curve $H(x)$, and the red curve is the actual removal amount distribution curve $h(x)$. The standard removal function has a maximum width of $2 R$ and a height of $A$. The discretization 
distance of the nodes is $L=R$. For each dwell node $X_{i}$, the dwell time function is set to $D_{i}=H\left(X_{\mathrm{i}}\right) / A$, which eliminates superposition coupling and is only a simple elementary algebraic operation. It shows that the algorithm has the same accuracy as the trapezoidal method of the one-dimensional definite integral problem, and its approximation residual is a second-order small quantity.

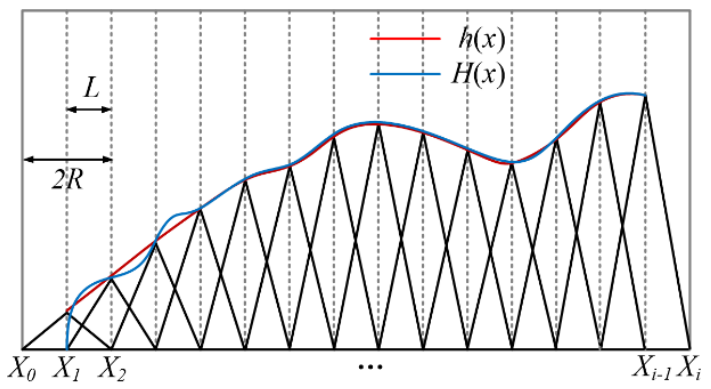

Figure 3. Elementary approximation solution for one dimension.

When $L=R$, the calculated residual error is already a second-order small quantity, but it can still be seen that the actual removal curve is not very smooth, which means that the smoothness of the optical processing surface is poor. When the discretization node is doubled and the spacing $L=R / 2$, then $D_{i}=H\left(X_{\mathrm{i}}\right) / 2 A$. The calculation principle of elementary geometric approximation for one-dimensional mesh refinement is shown in Figure 4 .

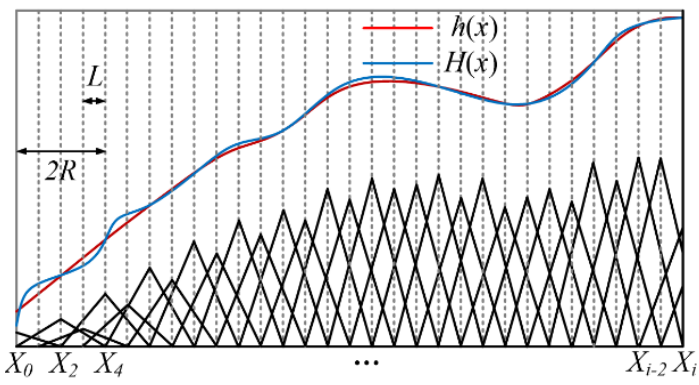

Figure 4. Mesh refinement principle for one dimension.

Generally, let $L=R / 2^{n}$, and the distribution function of target removal amount is $H(x)=2^{n} \cdot H(x) / 2^{n}$. The discretized node set $\left\{\mathrm{X}_{\mathrm{i}}\right\}$ is divided into $2^{n}$ groups. The node spacing in each group is $L=R$, and the phase difference between each group of nodes is an integer multiple of $R / 2^{n}$ in turn. Each set of nodes after partition is decoupled according to Figure 3 , and then $D_{i}=H\left(X_{i}\right) / 2^{n} / A$. In this way, the actual processing curve gradually becomes smooth.

According to the above analysis, the basic criteria for the elementary approximation of one-dimensional deconvolution are as follows:

1. It is acceptable to use an isosceles triangle as an approximate expression of the removal function in engineering;

2. The discretization distance of the nodes should not be more than half of the width of the removal function; otherwise, the deconvolution calculation will lose the ability to approximate;

3. When the node spacing is doubled, the time weight of each node is reduced by half, so the total time remains basically unchanged. The dwell time of the subdivided nodes is not the interpolation between the original discrete nodes, but the redistribution of the dwell time. The physical meaning is that the total removal amount is constant, and the removal function is constant, so the total time is basically conserved; 
4. The approximation residual of approximate solution is the same as that of definite integral trapezoid method, which is a second-order small quantity.

\subsubsection{Two-Dimensional Analysis}

The orthogonal grid $M$ is divided according to the spacing $R$. The coordinates of each grid node are $\left(x_{i}, y_{j}\right)$, and the target removal amount on the node is $\mathrm{H}\left(x_{i}, y_{j}\right)$. The plane distribution is discussed first, as shown in Figure 5.

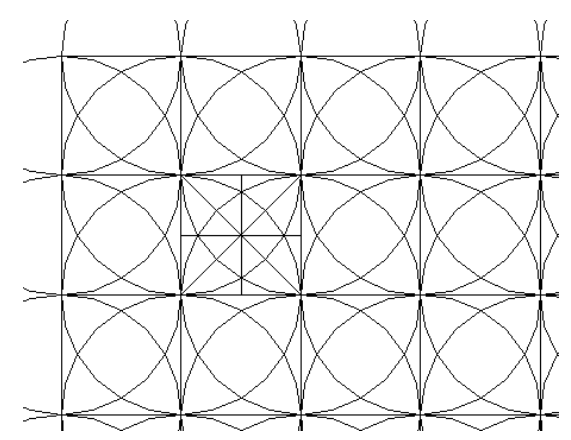

Figure 5. Mesh generation.

Let the removal function be a conic distribution and the center of the conic be the origin. The expression under the rectangular coordinate system is shown as follows:

$$
\left\{\begin{array}{l}
\varphi_{i j}(x, y)=\frac{A}{R}\left(R-\sqrt{\left(x-x_{i}\right)^{2}+\left(y-y_{j}\right)^{2}}\right),\left(x-x_{i}\right)^{2}+\left(y-y_{j}\right)^{2} \leq R^{2} \\
\varphi_{i j}(x, y)=0,\left(x-x_{i}\right)^{2}+\left(y-y_{j}\right)^{2}>R^{2}
\end{array}\right.
$$

Where $R$ is the radius of the circular support region of the removal function, and $A$ is the peak value of the removal function center. The expression in polar coordinate form is shown as follows:

$$
\left\{\begin{array}{c}
\varphi_{i j}(\rho, \theta)=\frac{A}{R}\left(R-\sqrt{\left(\rho \cos \theta-\rho_{i j} \cos \theta_{i j}\right)^{2}+\left(\rho \sin \theta-\rho_{i j} \sin \theta_{i j}\right)^{2}}\right), \\
\sqrt{\left(\rho \cos \theta-\rho_{i j} \cos \theta_{i j}\right)^{2}+\left(\rho \sin \theta-\rho_{i j} \sin \theta_{i j}\right)^{2}} \leq R \\
\varphi_{i j}(\rho, \theta)=0, \sqrt{\left(\rho \cos \theta-\rho_{i j} \cos \theta_{i j}\right)^{2}+\left(\rho \sin \theta-\rho_{i j} \sin \theta_{i j}\right)^{2}}>R
\end{array}\right.
$$

On the mesh, the removal function is simplified as follows:

$$
\left\{\begin{array}{l}
\varphi_{i}(x)=\frac{A}{R}\left(R-f a b s\left(x-x_{i}\right)\right),\left(x-x_{i}\right)^{2} \leq R^{2} \\
\varphi_{i}(x)=0,\left(x-x_{i}\right)^{2}>R^{2}
\end{array}\right.
$$

Or the following polar form:

$$
\left\{\begin{array}{l}
\varphi_{j}(y)=\frac{A}{R}\left(R-f a b s\left(y-y_{j}\right)\right),\left(y-y_{j}\right)^{2} \leq R^{2} \\
\varphi_{j}(y)=0,\left(y-y_{j}\right)^{2}>R^{2}
\end{array}\right.
$$

Where fabs represents the function of taking absolute value.

Suppose that each node $\left(x_{i}, y_{j}\right)$ of the grid is superimposed with a removal function $\varphi_{i j}$ of Equation (2) above, which has the same weight. Then, for the interior of the region $\mathrm{M}$, according to the symmetry, only the case of the middle region $R \times R$ needs to be considered. The edge of the whole grid area is special and will not be discussed here.

For the grid area, it is customary to take the lower left corner of the grid as the origin $\mathrm{O}$, so the center of the removal function is respectively located at the four corners of the 
grid, as shown in Figure 6. The green lines are auxiliary lines, the red lines are distances from each corner nodes, and $\mathrm{P}$ is an arbitrary point in the polishing area. Set the node number of $\mathrm{O}$ as $(i, j), \mathrm{OP}=r_{1}, \angle \mathrm{POC}=\theta_{1}, \mathrm{CP}=r_{2}, \angle \mathrm{PCB}=\theta_{2}, \mathrm{BP}=r_{3}, \angle \mathrm{PBA}=\theta_{3}, \mathrm{AP}=r_{4}$, and $\angle \mathrm{PAO}=\theta_{4}$ to give the following:

$$
\left\{\begin{array}{l}
r_{i j}=0, \theta_{i j}=0 \\
r_{i j+1}=R, \theta_{i j+1}=0 \\
r_{i+1 j+1}=\sqrt{2} R, \theta_{i+1 j+1}=\frac{\pi}{4} \\
r_{i+1 j}=R, \theta_{i+1 j}=\frac{\pi}{2}
\end{array}\right.
$$

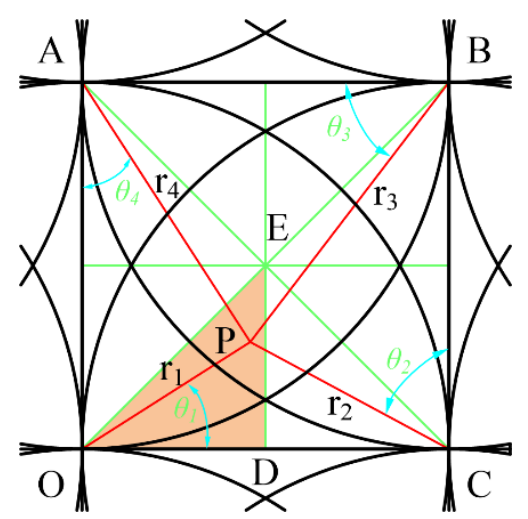

Figure 6. Superposition distribution map in grids.

According to Equation (3), there are the following:

$$
\left\{\begin{array}{l}
\varphi_{i j}\left(r_{1}, \theta_{1}\right)=\frac{A}{R}\left(R-r_{1}\right) \\
\varphi_{i j+1}\left(r_{2}, \theta_{2}\right)=\frac{A}{R}\left(R-r_{2}\right) \\
\varphi_{i+1 j+1}\left(r_{3}, \theta_{3}\right)=\frac{A}{R}\left(R-r_{3}\right) \\
\varphi_{i+1 j}\left(r_{4}, \theta_{4}\right)=\frac{A}{R}\left(R-r_{4}\right)
\end{array}\right.
$$

For a discrete mesh, due to the symmetry, only one-eighth of the triangular EOD area in the grid needs to be considered. This area can be further divided into four subareas: boundary line, M1, M2, and M3. For each subregion, only the value range of any point in the region under the function of each removal function (the maximum and minimum values) can be considered for evaluating the approximation ability of the approximate solution. The points where the maximum and minimum are located are the feature points in each square. According to the symmetry, these feature points must be obtained on the symmetry axis or the boundary of the square.

Similar to the one-dimensional case, let $L=R / 2^{n}$, and the distribution function of target removal amount is $H(x)=2^{n} \cdot H(x) / 2^{n}$. The discretized node set $\left(x_{i}, y_{j}\right)$ is divided into $2^{\mathrm{n}}$ groups. The node spacing in each group is $L=R$, and the phase difference between each group of nodes is an integer multiple of $R / 2^{n}$ in turn. Each set of nodes after partition is decoupled as above, and then there is $D_{i}=H\left(x_{i}, y_{j}\right) /\left(2^{n} A\right)$. The basic criteria for the primary approximation of two-dimensional deconvolution are as follows:

(1) It is acceptable to use cone as an approximate expression of the removal function in engineering.

(2) The distance of node discretization should not be larger than the radius of the removal function support domain; otherwise, the deconvolution calculation based on this method will lose the ability to approximate. 
(3) When the node spacing is doubled, the time weight of each node is reduced by half, so the total time remains basically unchanged.

(4) The approximation residual of the elementary geometric approximation method for two-dimensional deconvolution is completely acceptable compared with the actual polishing convergence rate.

\subsection{Dwell Time Algorithm Analysis}

2.3.1. Split Line Value Analysis

In the real polishing case, the workpiece surface is a two-dimensional planar. For each meridian segment or latitude segment, there are only two kernel functions. At this time, the superposition value of the removal function is always A, as shown in Figure 7.

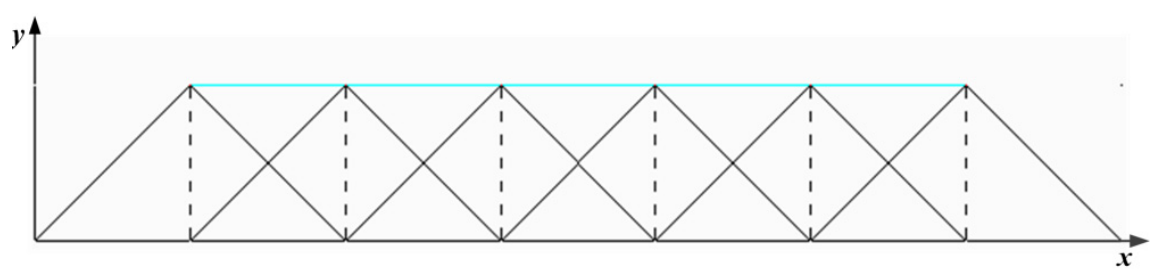

Figure 7. Schematic diagram of value taken on longitude and latitude line.

For example, let $x_{i}+1>x>x_{i}, x_{i+1}=x_{i}+R$, then the value on the grid is determined by Equation (8).

$$
f=\varphi_{i}(x)+\varphi_{i+1}(x)=\frac{A}{R}\left(R-\left(x-x_{i}\right)\right)+\frac{A}{R}\left(R-\left(x_{i+1}-x\right)\right)=A
$$

\subsubsection{Area M1 Value Analysis}

The point $\mathrm{P}$ is located in the region $\mathrm{M} 1$ and is acted on by four removal functions as shown in Figure 8.

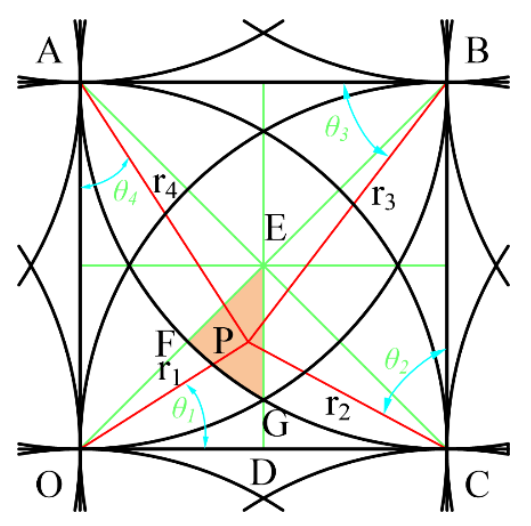

Figure 8. Four kernel function scopes.

According to Equations (3), (6) and (7), at this time the value of any point in M1 is determined by Equation (9).

$$
f=\varphi_{i j}\left(r_{1}, \theta_{1}\right)+\varphi_{i j+1}\left(r_{2}, \theta_{2}\right)+\varphi_{i+1 j+1}\left(r_{3}, \theta_{3}\right)+\varphi_{i+1 j}\left(r_{4}, \theta_{4}\right)=\frac{A}{R}\left(4 R-\left(r_{1}+r_{2}+r_{3}+r_{4}\right)\right)
$$


The relationships of each parameter are as follows:

$$
\begin{aligned}
& r_{2}=\sqrt{\left(r_{1} \cos \theta_{1}\right)^{2}+\left(R-r_{1} \cos \theta_{1}\right)^{2}}, \theta_{2}=\operatorname{Arcctg}\left(\frac{r_{1} \sin \theta_{1}}{R-r_{1} \cos \theta_{1}}\right) \\
& r_{3}=\sqrt{\left(r_{2} \cos \theta_{2}\right)^{2}+\left(R-r_{2} \cos \theta_{2}\right)^{2}}, \theta_{3}=\operatorname{Arcctg}\left(\frac{r_{2} \sin \theta_{2}}{R-r_{2} \cos \theta_{2}}\right) \\
& r_{4}=\sqrt{\left(r_{3} \cos \theta_{3}\right)^{2}+\left(R-r_{3} \cos \theta_{3}\right)^{2}}, \theta_{4}=\operatorname{Arcctg}\left(\frac{r_{3} \sin \theta_{3}}{R-r_{3} \cos \theta_{3}}\right) \\
& (\sqrt{2}-1) \mathrm{R} \leq r_{1} \leq \frac{\sqrt{2}}{2} R, \frac{5 \pi}{36} \leq \theta_{1} \leq \frac{\pi}{4}
\end{aligned}
$$

According to the symmetry, when point $P$ is located at the vertex $G$ of EFG, that is, $r_{3}=r_{4}=R, r_{1}=r_{2}=\sqrt{2-\sqrt{3}} R$, making the sum of $r_{1}+r_{2}+r_{3}+r_{4}$ the maximum, then the minimum value of the function in this area is:

$$
f_{\min }=2(1-\sqrt{2-\sqrt{3}}) A \approx 0.9647 A
$$

Since the sum of any two sides of a triangle is greater than the third side, it can be known that when $P$ is located at the center of the square; that is, $r_{1}=r_{2}=r_{3}=r_{4}=\sqrt{2} R / 2$, the sum of $r_{1}+r_{2}+r_{3}+r_{4}$, is a minimum, so the maximum value in this area is

$$
f_{\max }=4 \frac{A}{R}\left(R-\frac{\sqrt{2}}{2} R\right) \approx 1.172 A
$$

\subsubsection{Area M2 Value Analysis}

The point $\mathrm{P}$ is located in the region $\mathrm{M} 2$ and is acted on by three removal functions as shown in Figure 9.

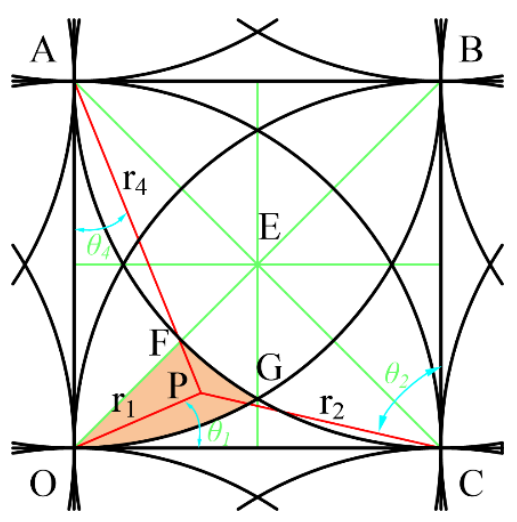

Figure 9. Three removal function scopes.

According to Equation (3), (6) and (7), at this time, the value of any point in M2 is determined by Equation (13).

$$
f=\varphi_{i j}\left(r_{1}, \theta_{1}\right)+\varphi_{i j+1}\left(r_{2}, \theta_{2}\right)+\varphi_{i+1 j}\left(r_{4}, \theta_{4}\right)=\frac{A}{R}\left(3 R-\left(r_{1}+r_{2}+r_{4}\right)\right)
$$


The relationships of each parameter are as follows:

$$
\begin{aligned}
& r_{2}=\sqrt{\left(r_{1} \cos \theta_{1}\right)^{2}+\left(R-r_{1} \cos \theta_{1}\right)^{2}}, \theta_{2}=\operatorname{Arcctg}\left(\frac{r_{1} \sin \theta_{1}}{R-r_{1} \cos \theta_{1}}\right) \\
& r_{3}=\sqrt{\left(r_{2} \cos \theta_{2}\right)^{2}+\left(R-r_{2} \cos \theta_{2}\right)^{2}}, \theta_{3}=\operatorname{Arcctg}\left(\frac{r_{2} \sin \theta_{2}}{R-r_{2} \cos \theta_{2}}\right) \\
& r_{4}=\sqrt{\left(r_{3} \cos \theta_{3}\right)^{2}+\left(R-r_{3} \cos \theta_{3}\right)^{2}}, \theta_{4}=\operatorname{Arcctg}\left(\frac{r_{3} \sin \theta_{3}}{R-r_{3} \cos \theta_{3}}\right) \\
& 0 \leq r_{1} \leq \sqrt{2-\sqrt{3}} R, 0 \leq \theta_{1} \leq \frac{\pi}{4}
\end{aligned}
$$

According to the symmetry, when point $P$ is located at the vertex $G$ of EFG, that is, $r_{4}=R, r_{1}=r_{2}=\sqrt{2-\sqrt{3}} R$, making the sum of $r_{1}+r_{2}+r_{4}$ the maximum, then the minimum value of the function in this area is:

$$
f_{\min }=2(1-\sqrt{2-\sqrt{3}}) A \approx 0.9647 A
$$

According to the symmetry, when point $P$ is located at the vertex $G$ of EFG, that is, $r_{1}=0, r_{2}=r_{4}=R$, making the sum of $r_{1}+r_{2}+r_{4}$ the minimum, then the maximum value in this area is:

$$
f_{\max }=A
$$

\subsubsection{Area M3 Value Analysis}

The point $\mathrm{P}$ is located in the region M2 and is acted by two removal functions as shown in Figure 10.

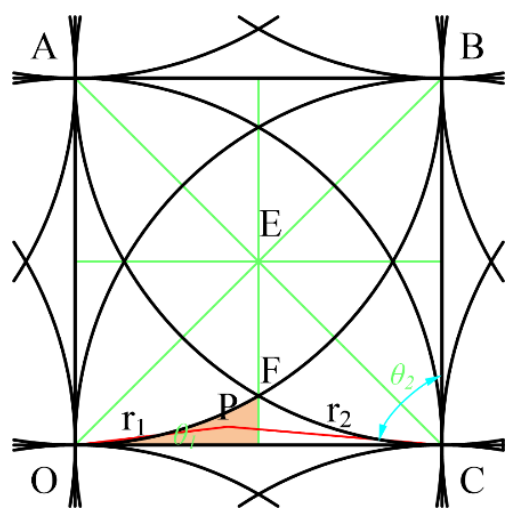

Figure 10. Two removal function scopes.

According to Equation (3), (6) and (7), at this time, the value of any point in M3 is determined by Equation (17).

$$
f=\varphi_{i j}\left(r_{1}, \theta_{1}\right)+\varphi_{i j+1}\left(r_{2}, \theta_{2}\right)=\frac{A}{R}\left(2 R-\left(r_{1}+r_{2}\right)\right)
$$

The relationships of each parameter are as follows:

$$
\begin{aligned}
& r_{2}=\sqrt{\left(r_{1} \cos \theta_{1}\right)^{2}+\left(R-r_{1} \cos \theta_{1}\right)^{2}}, \theta_{2}=\operatorname{Arcctg}\left(\frac{r_{1} \sin \theta_{1}}{R-r_{1} \cos \theta_{1}}\right) \\
& 0 \leq r_{1} \leq \sqrt{2-\sqrt{3}} R, 0 \leq \theta_{1} \leq \frac{5 \pi}{36}
\end{aligned}
$$


According to the geometric relationship and symmetry, when point $\mathrm{P}$ is located at the vertex $G$ of a curved triangle, that is, $r_{1}=r_{2}=\sqrt{2-\sqrt{3}} R$, the sum of $r_{1}+r_{2}$ is the maximum, then the following function obtains the minimum value:

$$
f_{\min }=2(1-\sqrt{2-\sqrt{3}}) \mathrm{A} \approx 0.9647 A
$$

When point $\mathrm{P}$ is located on the side of the curved triangle $\mathrm{OD}$, that is, $r_{1}+r_{2}=R$, the sum of $r_{1}+r_{2}$ is the smallest, and the function obtains the maximum value:

$$
f_{\max }=A
$$

\subsubsection{Numerical analysis}

Figure 11 is a cloud picture of simulation calculation of an equal weight superposition distribution of removal function in the middle area. The distribution characteristics are consistent with the theoretical analysis.

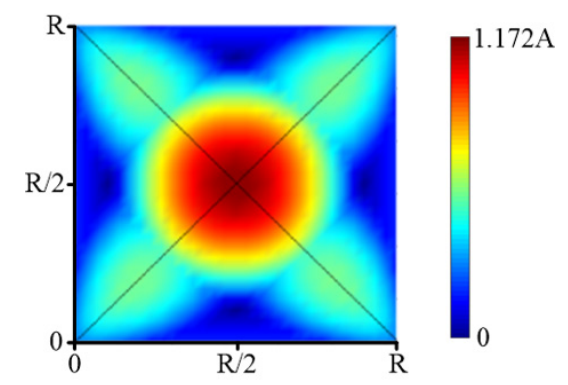

Figure 11. Simulation results of removal function superposition.

According to the results of the above analysis, on the whole grid the maximum and minimum values of the weight superposition distribution of the removal function are as follows:

$$
\begin{aligned}
& f_{\min }=2(1-\sqrt{2-\sqrt{3}}) A \approx 0.9647 A \\
& f_{\max }=4\left(1-\frac{\sqrt{2}}{2}\right) A \approx 1.172 A
\end{aligned}
$$

This reflects the approximation ability of the conic distribution removal function to the plane, that is, the error level based on the elementary approximation method. The convergence accuracy of the calculation is much higher than the actual polishing convergence rate.

More generally, if each removal function is weighted according to the value of the surface distribution at the center, the conical distribution removal function can approximate the general surface better. That is to say, for any initial error distribution, the dwell time function the kernel of which is a cone distribution can be approximately determined by the weight of the kernel's center. At this time, the higher deconvolution problem can be simplified to a basic function-value calculation problem.

\section{Simulations}

The example of a two-dimensional deconvolution based on an elementary approximation is shown below. Three sets of simulations use the cone distribution removal function, and the removal amount distribution is a plane, sphere and arbitrary surface, respectively. The dwell time function is solved by the elementary approximation method proposed in this paper. The results are shown in Figures 12-14. 


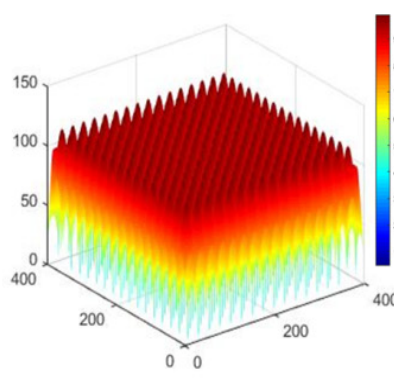

(a)

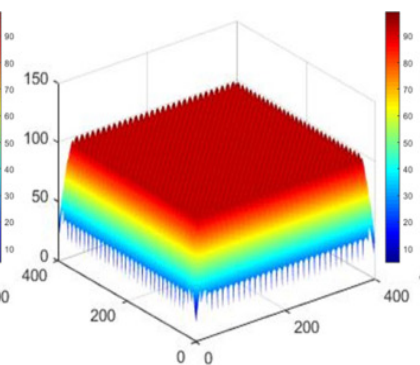

(b)

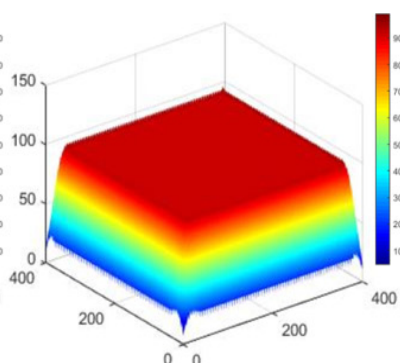

(c)

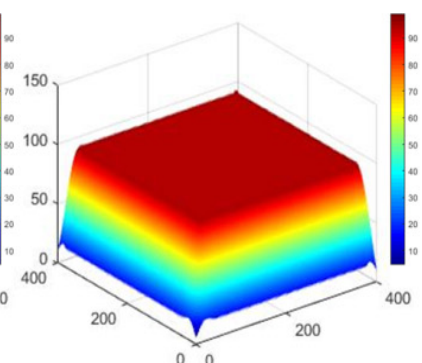

(d)

Figure 12. Approximation results for the distribution of plane removal amount (a) Grid spacing $L=R$, (b) Grid spacing $\mathrm{L}=\mathrm{R} / 2$, (c) Grid spacing $\mathrm{L}=\mathrm{R} / 4$, (d) Ideal plan distribution.

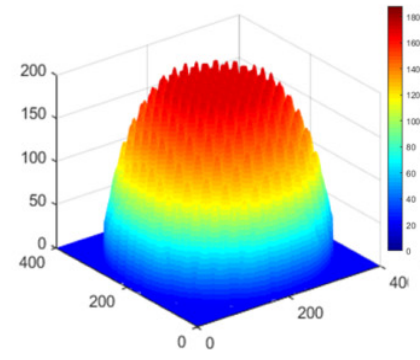

(a)

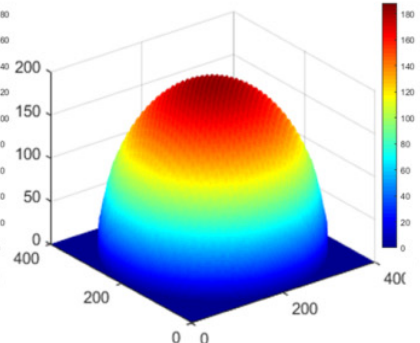

(b)

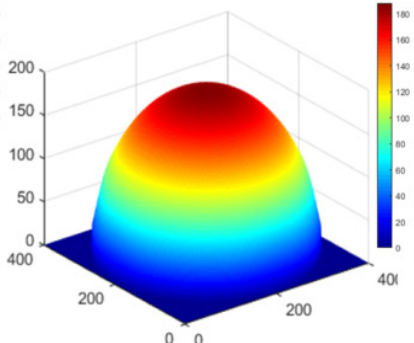

(c)

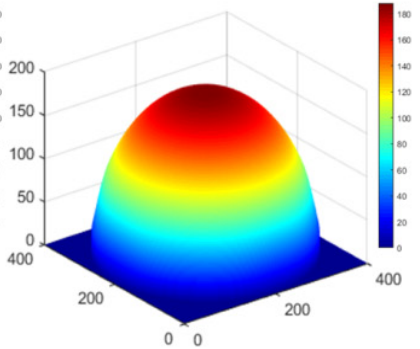

(d)

Figure 13. Approximation results for the distribution of spherical removal (a) Grid spacing $L=R$, (b) Grid spacing $L=R / 2$, (c) Grid spacing $L=R / 4$, (d) Ideal hemispheric distribution.

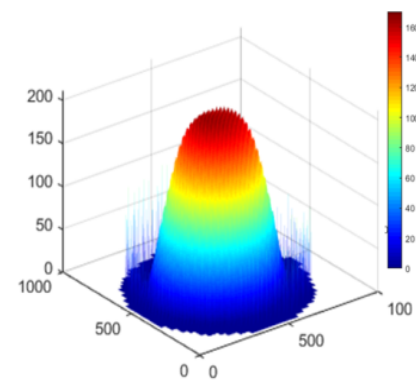

(a)

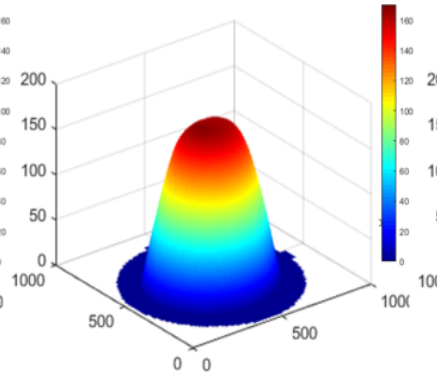

(b)

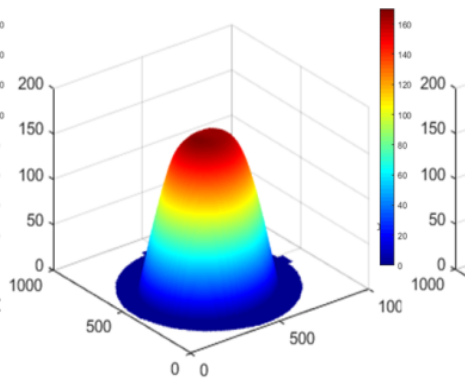

(c)

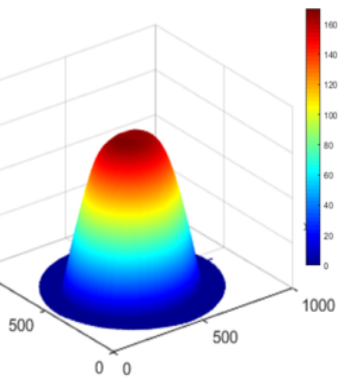

(d)

Figure 14. Approximation results for the distribution of arbitrary removal (a) Grid spacing $L=R,(\mathbf{b})$ Grid $\operatorname{spacing} L=R / 2$, (c) Grid spacing $L=R / 4$, (d) Original surface distribution.

Apparently, the numerical simulation results of a deconvolution calculation based on an elementary approximation are satisfactory.

\section{Results and Discussion}

\subsection{Experiment Setup and the Parameters}

To validate the effectiveness of the proposed algorithm, repetitious experimental studies on flatness figuring was carried out based on the self-developed ion-beam figuring (IBF) machine. The experiment setup is shown in Figure 15. It can process planar, spherical and aspheric parts with a maximum size of $300 \mathrm{~mm} \times 300 \mathrm{~mm}$, and the positioning accuracy of the linear axis is below $0.005 \mathrm{~mm}$. This machine can be used for the corresponding 
experimental verification of the dwell time solving algorithm. The specific parameters of the polishing processing are shown in Table 1.

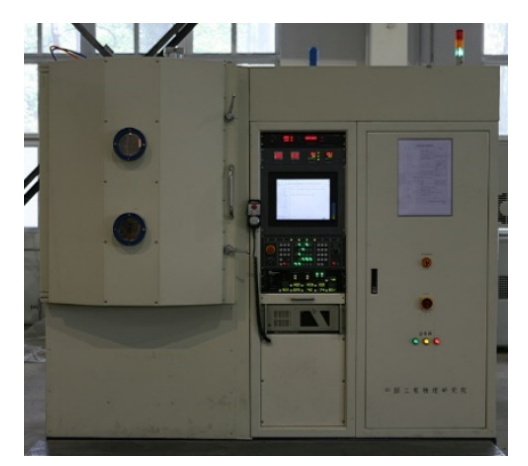

Figure 15. The self-developed IBF machine.

Table 1. Parameters of the polishing process.

\begin{tabular}{cc}
\hline Parameter & Value \\
\hline Ion beam voltage & $800 \mathrm{~V}$ \\
Ion beam current & $60 \mathrm{~mA}$ \\
Ion beam Angle & $0^{\circ}$ \\
Processing distance & $150 \mathrm{~mm}$ \\
Grating spacing & $2 \mathrm{~mm}$ \\
\hline
\end{tabular}

IBF spots were taken on the IBF machine, and the results are shown in Figure 16. The polishing result was detected by the INF300H-LP-WM interferometer made in China, with RMS repeatability of less than $0.3 \mathrm{~nm}$. The removal function result of IBF, shown in Figure 16, was tested on a fixed point, and polishing time was $60 \mathrm{~s}$. The peak removal rate (PRR) of influence function was $0.30614 \lambda / \mathrm{min}$, where $\lambda$ was $658 \mathrm{~nm}$, and the volume removal rate (VRR) was $0.056474 \mathrm{~mm}^{3} / \mathrm{min}$.

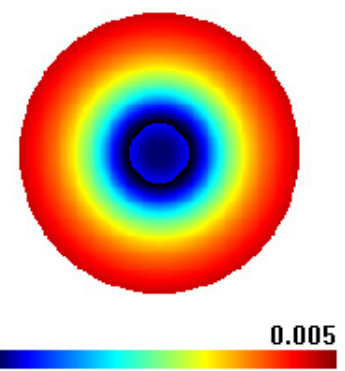

(a)

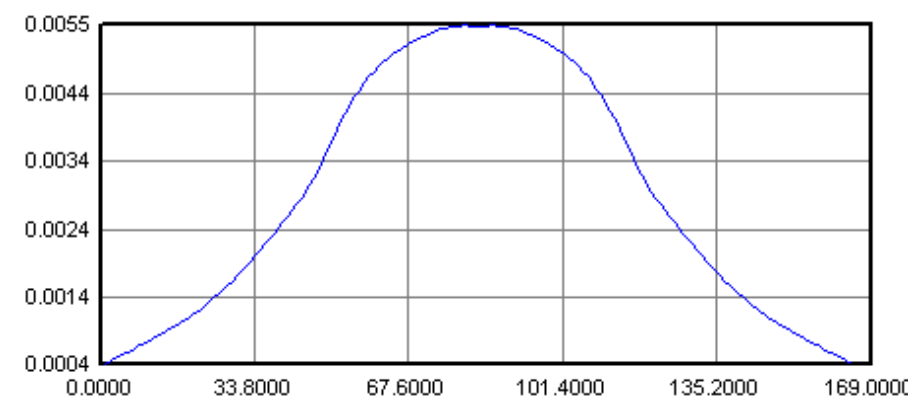

(b)

Figure 16. Removal function in case 1: (a) surface map, (b) surface profile.

\subsection{Results and Discussion}

\subsubsection{Experiment Case 1}

The workpiece used for figuring is silica flat and the diameter is $\varphi 120 \mathrm{~mm}$. The scanning path is in raster with a step of $1.5 \mathrm{~mm}$ and spacing of $3.0 \mathrm{~mm}$. Initial flatness was PV $0.259 \lambda$, RMS $0.050 \lambda$; Predicted flatness was PV $0.051 \lambda$ and RMS $0.004 \lambda$, with a convergence rate of $80.0 \%$ and $92 \%$, respectively.

Experimental result indicated that full-aperture machined flatness was PV $0.077 \lambda$ and RMS $0.013 \lambda$ with a convergence rate of $70.3 \%$ and $74.5 \%$ respectively, where the missing 
data in the periphery was caused by the fixture. The aperture machined flatness of $90 \%$ was PV $0.063 \lambda$ and RMS $0.012 \lambda$ with a convergence rate of $64 \%$ and $70 \%$ respectively, as shown in Figures 17 and 18.

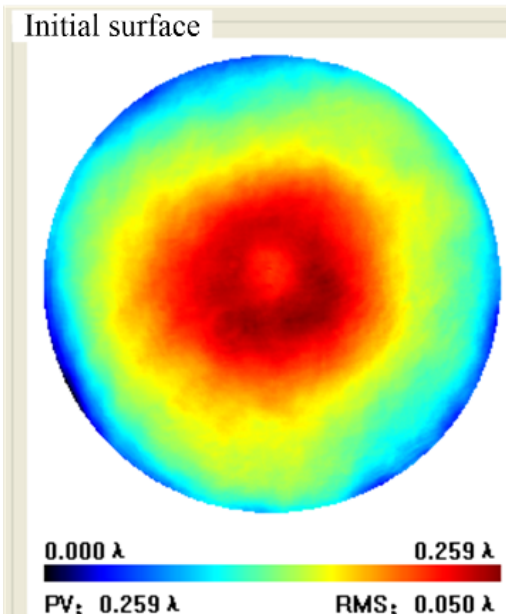

(a)

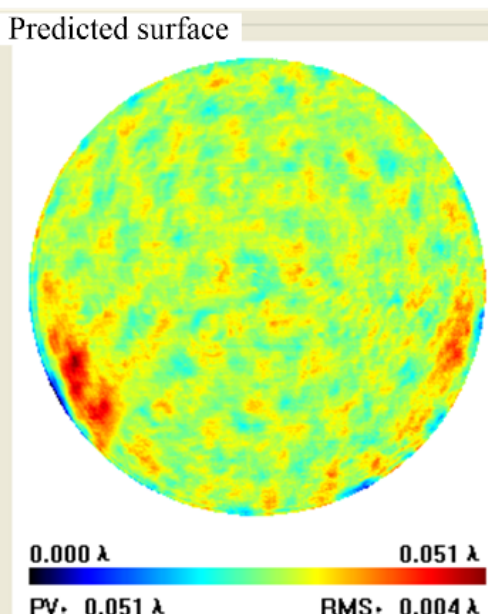

(b)

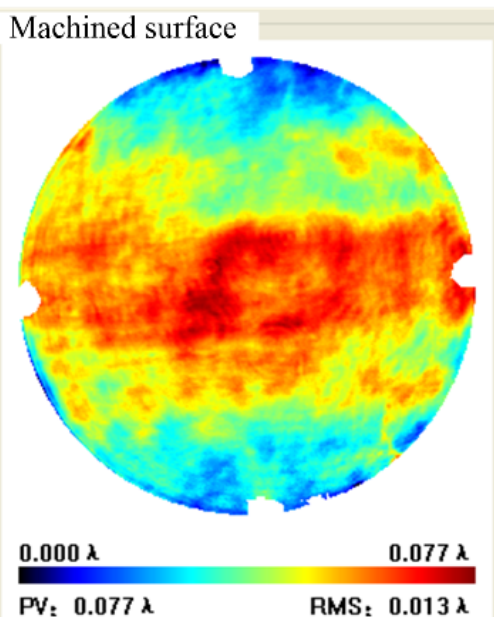

(c)

Figure 17. Full aperture data in case 1: (a) Initial surface, (b) Predicted surface, (c) Machined surface.

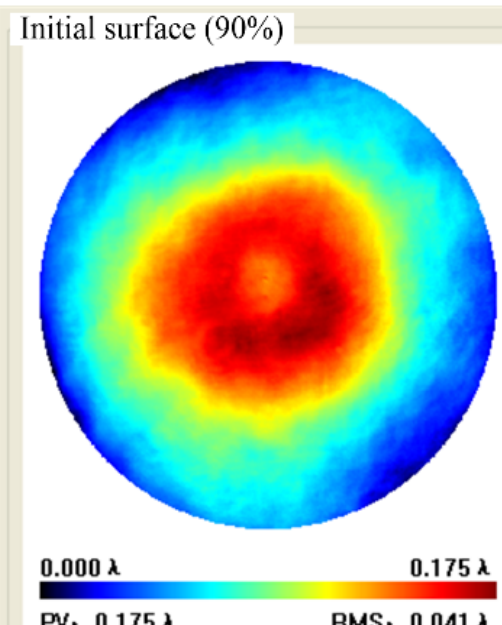

(a)

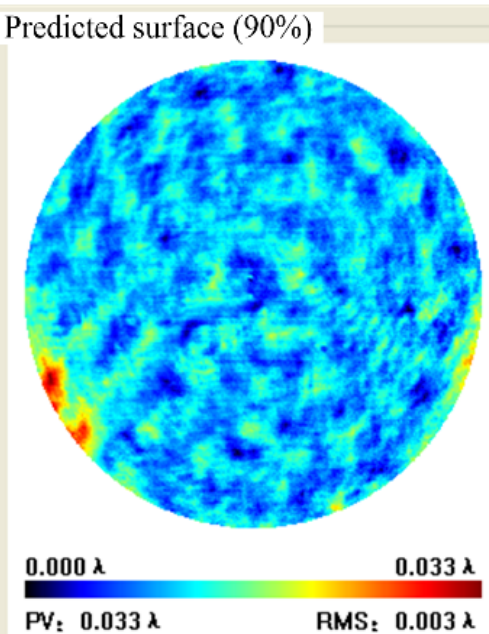

(b)

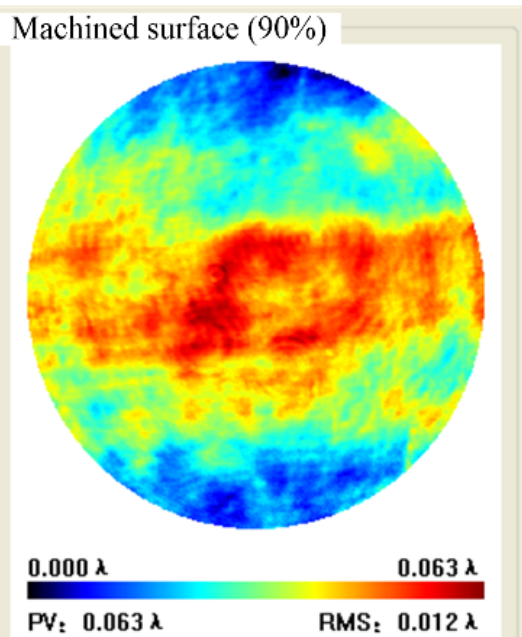

(c)

Figure 18. Case 1 90\% aperture data: (a) Initial surface, (b) Predicted surface, (c) Machined surface.

\subsubsection{Experiment Case 2}

The universal measuring software was employed. The PRR of influence function was $0.244 \lambda / \mathrm{min}$, and the VRR was $0.0098 \mathrm{~mm}^{3} / \mathrm{min}$ as shown in Figure 19.

The workpiece is fused silica with diameter of $\varphi 170 \mathrm{~mm}$. Surface map is measured using the relative accuracy method. Initial flatness of $\varphi 150 \mathrm{~mm}$ aperture is $P V_{\varphi 150}=0.048$ $\lambda, \operatorname{RMS}_{\varphi 150}=0.008 \lambda$, and those of $\varphi 100 \mathrm{~mm}$ aperture are $\mathrm{PV}_{\varphi 100}=0.030 \lambda, \mathrm{RMS}_{\varphi 100}=$ $0.007 \lambda$ respectively, as shown in Figure 20. 

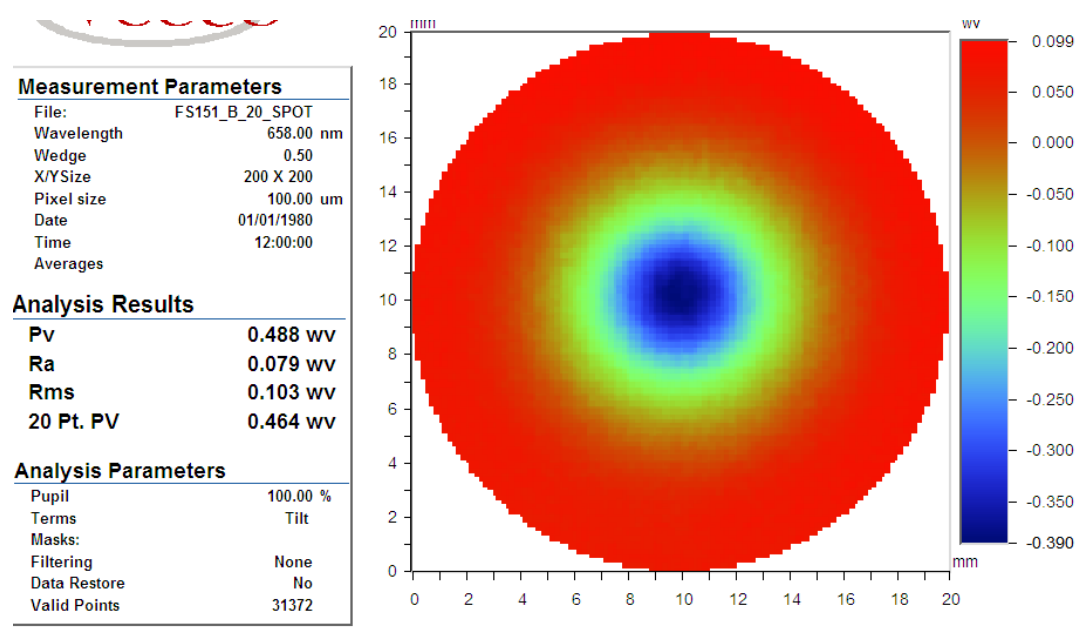

Figure 19. Removal function in case 2.

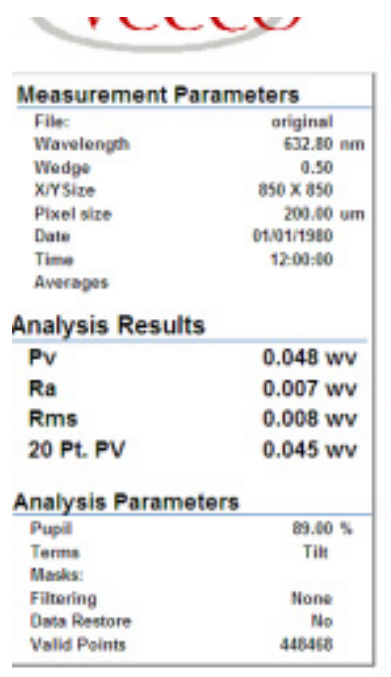

vしLU

\begin{tabular}{|c|c|}
\hline \multicolumn{2}{|c|}{ Measurement Parameters } \\
\hline File: & orighnal \\
\hline Wawolength & $552.00 \mathrm{~nm}$ \\
\hline $\begin{array}{l}\text { Wedge } \\
\text { xyseres }\end{array}$ & 0.50 \\
\hline Xirsite & $850 \times 850$ \\
\hline $\begin{array}{l}\text { Pixel size } \\
\text { Date }\end{array}$ & 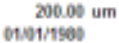 \\
\hline $\operatorname{Tim}$ & 1200.00 \\
\hline Awsrages & \\
\hline \multicolumn{2}{|c|}{ Analysis Results } \\
\hline Pv & $0.030 \mathrm{wv}$ \\
\hline Ra & $0.006 \mathrm{wv}$ \\
\hline Rms & $0.007 \mathrm{wv}$ \\
\hline $20 \mathrm{Pt} . \mathrm{PV}$ & $0.029 \mathrm{wv}$ \\
\hline \multicolumn{2}{|c|}{ Analysis Parameters } \\
\hline & 58.805 \\
\hline & Tilit \\
\hline \multicolumn{2}{|l|}{ Masks: } \\
\hline Filtaring & Hone \\
\hline Dona Ressore & $\begin{aligned} N o \\
1070\end{aligned}$ \\
\hline Valid Poines & 195712 \\
\hline
\end{tabular}

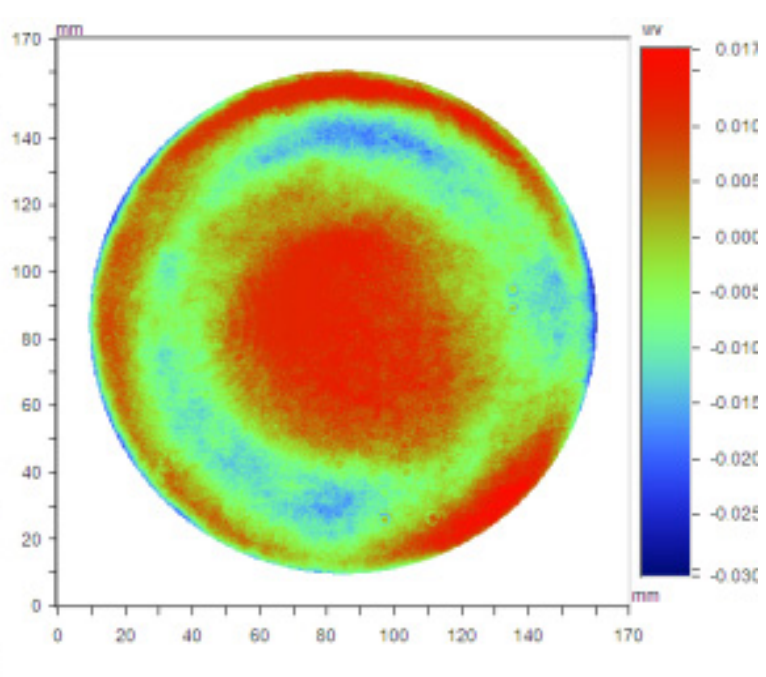

(a)

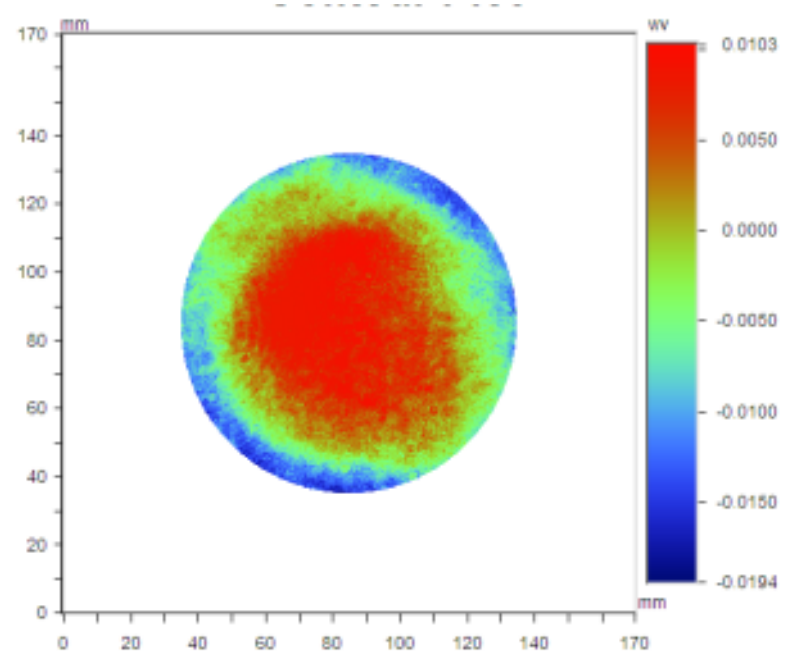

(b)

Figure 20. Initial surface in case 2: (a) $\varphi 150 \mathrm{~mm}$ aperture, (b) $\varphi 100 \mathrm{~mm}$ aperture. 
The scanning path was rasterized with $2 \mathrm{~mm}$ spacing, and the predicted machine time was $83.8 \mathrm{~min}$; the predicted flatness of $\varphi 150 \mathrm{~mm}$ aperture was $\mathrm{PV}_{\mathrm{c} 150}=0.025 \lambda$, RMS $_{\mathrm{c} 150}=0.003 \lambda$; and those of $\varphi 100 \mathrm{~mm}$ were $\mathrm{PV}_{\mathrm{c} 100}=0.014 \lambda ; \mathrm{RMS}_{\mathrm{c} 100}=0.002 \lambda$, as illustrated in Figure 21.

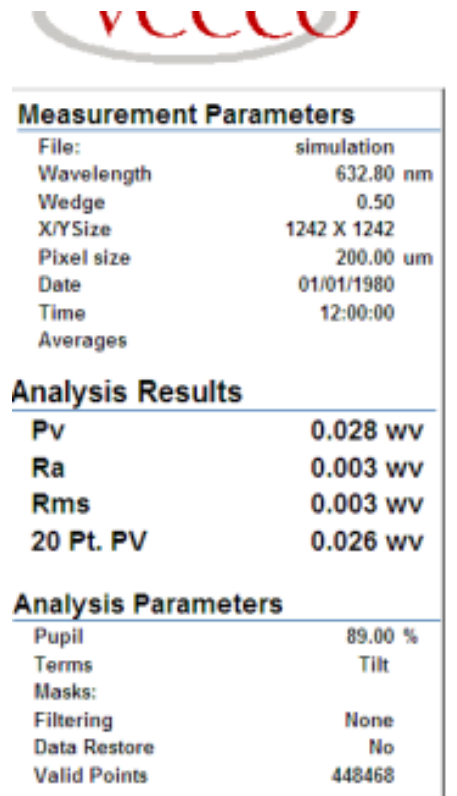

\section{VCCLU}

\section{Measurement Parameters}

Fle:

Wavelength

Wedge

$\mathrm{X} / \mathrm{YSI} 2 \mathrm{O}$

PA:el $₫ 28$

Date

Thme

Average:

Analysis Results

Pv

Ra

Rms

20 Pt. PV

Analysis Parameters

\begin{tabular}{lr}
\hline Pupll & $58.80 \%$ \\
Term : & TIt \\
Masks: & Analysis Mask \\
Fitering & None \\
Deta Restore & No \\
Valld Polnt: & 195671 \\
\hline
\end{tabular}

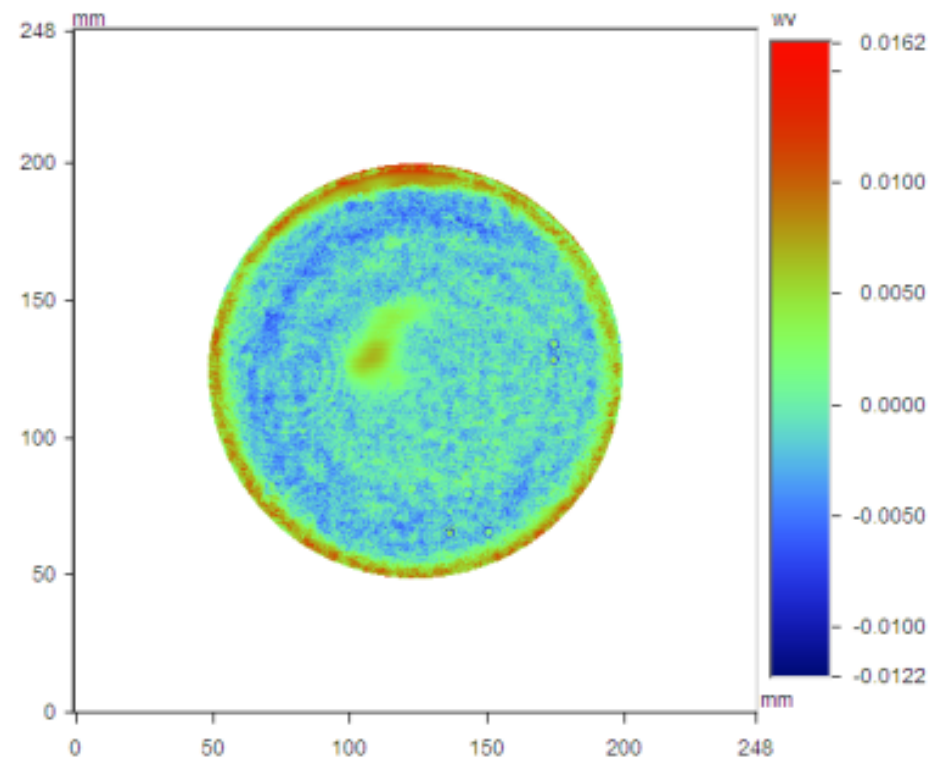

(a)

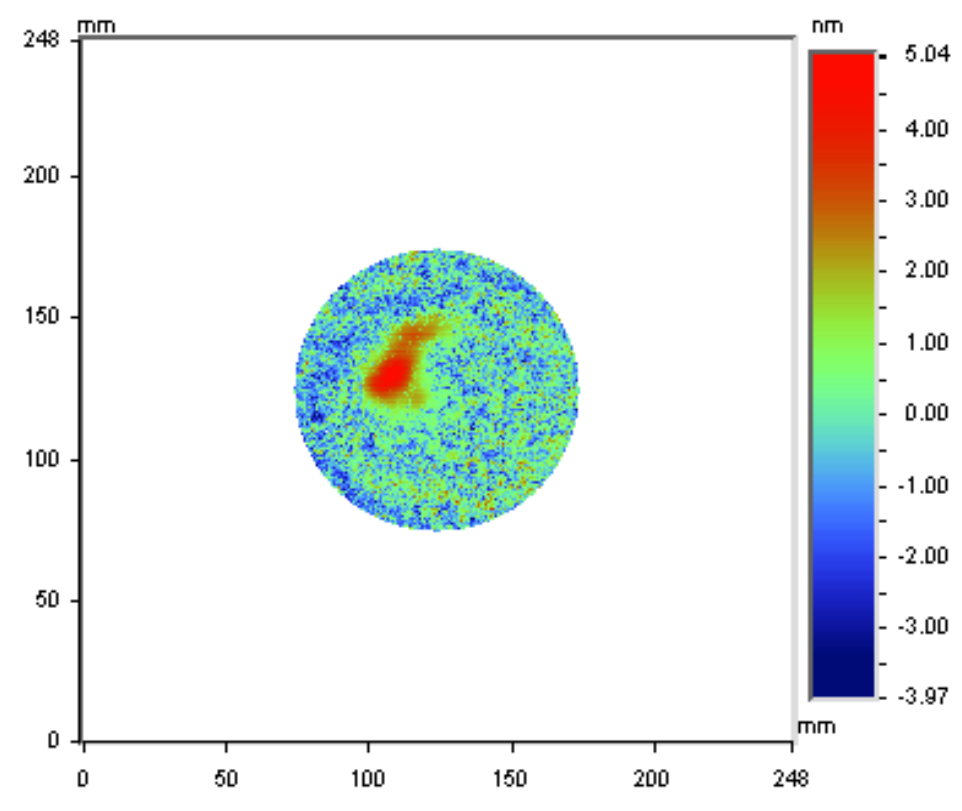

(b)

Figure 21. Predicted surface in case 2 (a) $\varphi 150 \mathrm{~mm}$ aperture (b) $\varphi 100 \mathrm{~mm}$ aperture.

The practical polishing time was $84 \mathrm{~min}$. The surface map was also measured using the relative accuracy method. The polished flatness of the $\varphi 150 \mathrm{~mm}$ aperture was $\mathrm{PV}_{\mathrm{r} 150}=0.028 \lambda$, and $\mathrm{RMS}_{\mathrm{r} 150}=0.005 \lambda$, and those of $\varphi 100 \mathrm{~mm}$ were $\mathrm{PV}_{\mathrm{cr} 100}=0.014 \lambda$, and $\mathrm{RMS}_{\mathrm{c} 100}=0.002 \lambda$, as shown in Figure 22 . 

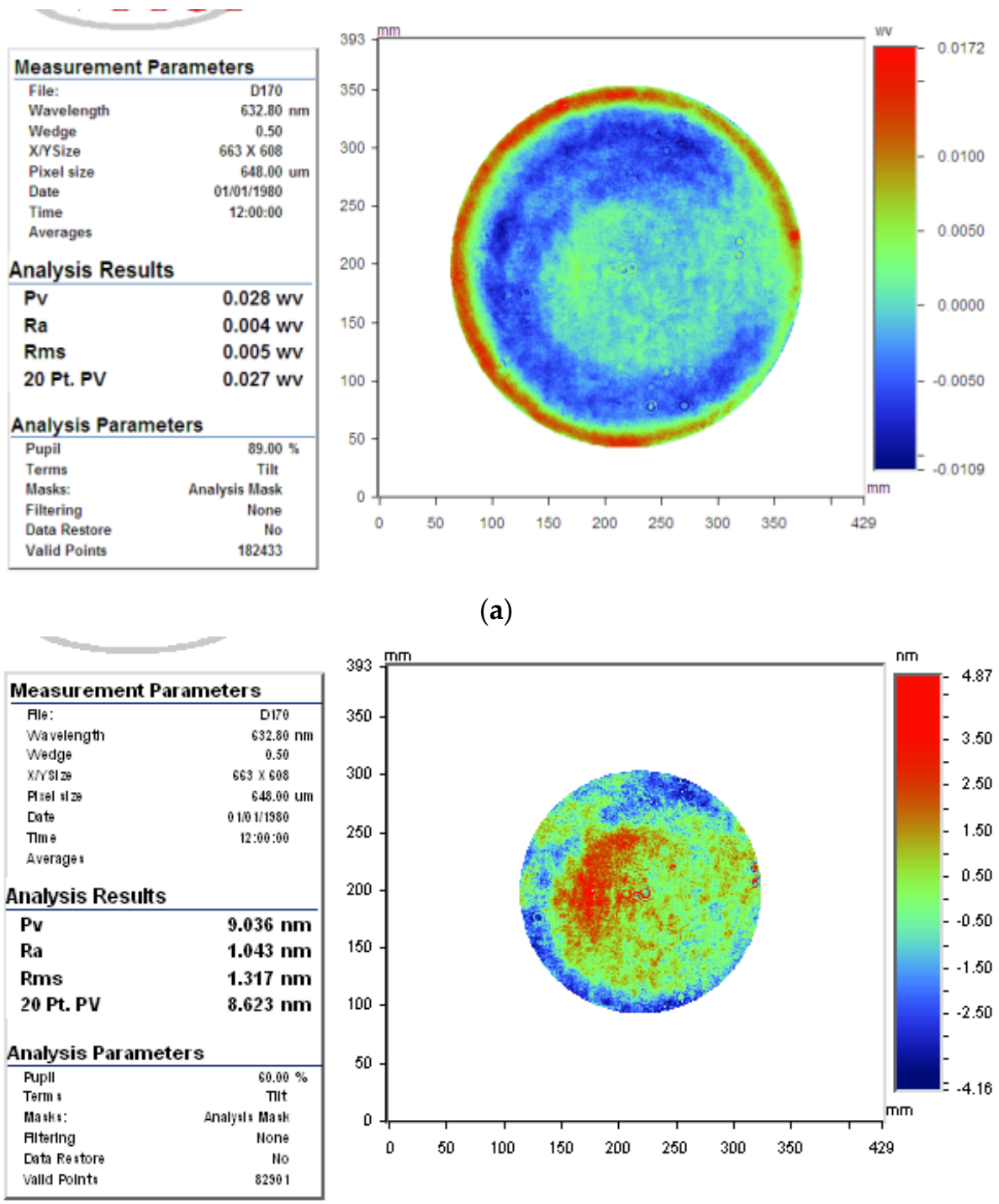

(a)

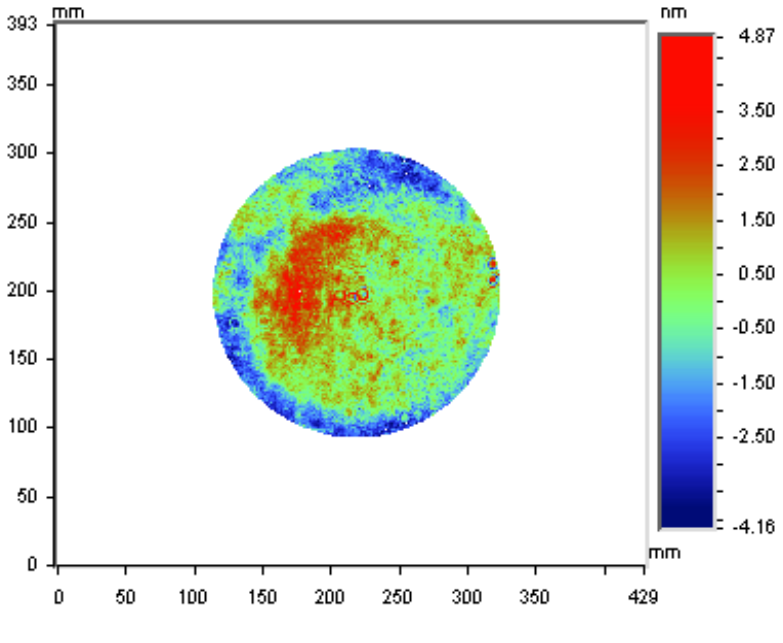

(b)

Figure 22. Polished surface in case 2: (a) $\varphi 150 \mathrm{~mm}$ aperture, (b) $\varphi 100 \mathrm{~mm}$ aperture.

Simulated convergence rate was

$$
\eta_{c 150}=\frac{p v_{o 150}-p v_{c 150}}{p v_{0150}} \times 100 \%=47.9 \%
$$

And the practical convergence rate was

$$
\eta_{r 150}=\frac{p v_{r 150}-p v_{r 150}}{p v_{r 150}} \times 100 \%=41.7 \%
$$

Taking the surface PV value of $\varphi 100 \mathrm{~mm}$ aperture as the evaluation index, the predicted convergence rate was $52.0 \%$, and the practical convergence rate was $51.9 \%$.

\section{Conclusions}

In this paper, an elementary approximation of the dwell time algorithm for singlepeak rotational symmetry removal function was presented. The work showed that it is engineeringly acceptable to use the cone distribution as the removal function to approximate expressions of all the subaperture polishing with a single-peak rotational symmetry removal function, such as CCOS, IBF, or BP. The dwell time algorithm model and compu- 
tation method were given. When the distance of node discretization was not more than the radius of the support domain of removal function, the coupling characteristic of the deconvolution problem could be eliminated by using the elementary approximation solution proposed in this paper. Theoretical analysis, numerical simulation and experimental results show that the proposed method had a lower calculated residual error than the initial value by one order of magnitude, and had a higher approximation ability. The flatness of $\varphi 150 \mathrm{~mm}$ and $\varphi 100 \mathrm{~mm}$ workpieces achieved $\mathrm{PVr}_{150}=0.028 \lambda$ and $\mathrm{PVcr}_{100}=0.014 \lambda$, respectively.

In contrast to conventional dwell time algorithms, this work transformed the superposition and coupling features of the deconvolution problem into a simple calculation of the discretization function value. When the discrete nodes were doubled, the time weight of each node was then halved; consequently, the total time remained unchanged. The approximation ability or smoothness of the deconvolution result was greatly increased, which agreed with the engineering reality that total polishing time remains unchanged if the total removal amount and removal function are unchanged. Compared with conventional methods, the proposed algorithm has obvious advantages for improving calculation efficiency and smoothness, which is of great significance for the efficient computation of large-aperture optical polishing.

Nevertheless, the calculation accuracy of the proposed dwell time algorithm is related to the symmetry of the removal function and its approximation error. Meanwhile, the dwell time algorithm has a limited ability to correct mid-to-high frequency errors determined by the sampling characteristics of the discretization nodes and the scale of the removal function. The approximation error in the edge area of the workpiece needs further analysis.

Author Contributions: All authors have contributed to the study conception and design. Y.W. designed the experiments and wrote the paper. Y.Z. performed the experiments and analyzed the data. R.K. and F.J. supervised the whole project and gave advices on manuscript editing. All authors read and approved the final manuscript.

Funding: This research was funded by the National Natural Science Foundation of China (No. 52005463); the Science Challenge Project (No.TZ2016006-0504-01); High-end CNC Machine Tools and Basic Manufacturing Equipment Major National Science and Technology Project (2017ZX04022001); Laboratory of Precision Manufacturing Technology, CAEP.

Data Availability Statement: NOMENCLATURE is contained within the main text.

Acknowledgments: The authors thank the technical support from peer fellows.

Conflicts of Interest: The authors declare no conflict of interest.

\section{Nomenclature}

$\begin{array}{ll}\mathrm{R}(\mathrm{x}, \mathrm{y}) & \text { Removal function } \\ \mathrm{D}(\mathrm{x}, \mathrm{y}) & \text { Dwell time function } \\ \mathrm{H}(\mathrm{Xi}, \mathrm{y}) & \text { Target removal amount } \\ \mathrm{A} & \text { Peak value of the removal function center } \\ \mathrm{fabs} & \text { Function of taking absolute value } \\ \mathrm{t}(\mathrm{xi}, \mathrm{yi}) & \text { Dwell time at the } \mathrm{i} \text { th path node } \\ \mathrm{h}(\mathrm{xk}, \mathrm{yk}) & \text { Desired amount of removed material at the } \mathrm{k} \text { th figure-control node } \\ \mathrm{Nt} & \text { Total number of the path nodes } \\ \mathrm{Nh} & \text { Total number of the figure-control nodes } \\ \mathrm{r}(\mathrm{xk}-\mathrm{xi}, \mathrm{yk}-\mathrm{yi}) & \text { Amount of removed material at the } \mathrm{k} \text { th figure-control node } \\ \mathrm{L} & \text { Discretization distance of nodes } \\ \mathrm{CCOS} & \text { Computer controlled optical surfacing } \\ \mathrm{MRF} & \text { Magnetorheological finishing } \\ \mathrm{IBF} & \text { Ion beam figuring } \\ \mathrm{BP} & \text { Bonnet polishing }\end{array}$




\section{References}

1. Zhao, Q.Z.; Zhang, L.; Han, Y.J.; Fan, C. Polishing path generation for physical uniform coverage of the aspheric surface based on the Archimedes spiral in bonnet polishing. Proc. Inst. Mech. Eng. B J. Eng. Manuf. 2019, 233, 2251-2263. [CrossRef]

2. Zhao, Q.Z.; Zhang, L.; Fan, C. Six-directional pseudorandom consecutive unicursal polishing path for suppressing mid-spatial frequency error and realizing consecutive uniform coverage. Appl. Opt. 2019, 58, 8529-8541. [CrossRef] [PubMed]

3. Aspden, R.; Mcdonough, R.; Nitchie, F.R. Computer assisted optical surfacing. Appl. Opt. 1972, 11, 2739-2747. [CrossRef] [PubMed]

4. Jones, R.A. Optimization of computer controlled polishing. Appl. Opt. 1977, 16, 218-224. [CrossRef] [PubMed]

5. Carnal, C.L.; Egert, C.M.; Hylton, K.W. Advanced matrix-based algorithm for ion-beam milling of optical components. In Proceedings of the Current Developments in Optical Design and Optical Engineering II, SPIE, San Diego, CA, USA, 20-21 July 1992; Volume 1752, pp. 54-62.

6. Drueding, T.W.; Fawcett, S.C.; Wilson, S.R.; Bifano, T.G. Ion beam figuring of small optical components. Opt. Eng. 1995, $34,3565$.

7. Waluschka, E. Cylindrical optic figuring dwell time optimization. In Proceedings of the International Symposium on Optical Science and Technology, SPIE, San Diego, CA, USA, 30-31 July 2000; Volume 4138, pp. 25-32.

8. Shanbhag, P.M.; Feinberg, M.R.; Sandri, G.; Horenstein, M.N.; Bifana, T.G. Ion-beam machining of millimeter scale optics. Appl. Opt. 2000, 39, 599-611. [CrossRef] [PubMed]

9. Zheng, L.G.; Zhang, X.J. A novel resistance iterative algorithm for CCOS. In Proceedings of the SPIE-The International Society for Optical Engineering, San Diego, CA, USA, 14 August 2006; Volume 6288, p. 62880N-1-9.

10. Zhou, L.; Dai, Y.F.; Xie, X.H.; Jiao, C.J.; Li, S.Y. Model and method to determine dwell time in ion beam figuring. Nanotechnol. Precis. Eng. 2007, 5, 107-112.

11. Wu, J.F.; Lu, Z.W.; Zhang, H.X.; Wang, T.S. Dwell time algorithm in ion beam figuring. Appl. Opt. 2009, 48, 3930-3937. [CrossRef] [PubMed]

12. Jiao, C.J.; Li, S.Y.; Xie, X. Algorithm for ion beam figuring of low-gradient mirrors. Appl. Opt. 2009, 48, 4090-4096. [CrossRef] [PubMed]

13. Bo, J.; Zhen, M. Dwell time method based on richardson-lucy algorithm. In Proceedings of the Space Optics and Earth Imaging and Space Navigation, SPIE, Beijing, China, 4-6 June 2017; Volume 10463.

14. Guo, J. Corrective finishing of a micro-aspheric mold made of tungsten carbide to $50 \mathrm{~nm}$ accuracy. Appl. Opt. 2015, 54, 5764-5770. [CrossRef] [PubMed]

15. Pan, R.; Zhang, Y.J.; Ding, J.B.; Cao, C.; Wang, Z.Z.; Jiang, T.; Peng, Y.F. Rationality optimization of tool path spacing based on dwell time calculation algorithm. Int. J. Adv. Manuf. Technol. 2016, 84, 2055-2065. [CrossRef]

16. Li, L.X.; Xue, D.L.; Deng, W.J.; Wang, X.; Bai, Y.; Zhang, F.; Zhang, X.J. Positive dwell time algorithm with minimum equal extra material removal in deterministic optical surfacing technology. Appl. Opt. 2017, 56, 9098-9104. [CrossRef] [PubMed]

17. Li, Y.; Zhou, L. Solution algorithm of dwell time in slope-based figuring model. In Proceedings of the AOPC 2017: Optoelectronics and Micro/Nano-Optics, SPIE, Beijing, China, 4-6 June 2017; Volume 10460.

18. Wang, Q.H.; Liang, Y.J.; Xu, C.Y.; Li, J.R.; Zhou, X.F. Generation of material removal map for freeform surface polishing with tilted polishing disk. Int. J. Adv. Manuf. Technol. 2019, 102, 4213-4226. [CrossRef]

19. Wan, S.L.; Zhang, X.C.; Wang, W.; Xu, M. Effect of pad wear on tool influence function in robotic polishing of large optics. Int. J. Adv. Manuf. Technol. 2019, 102, 2521-2530. [CrossRef]

20. Cao, Z.C.; Cheung, C.F.; Ho, L.T.; Liu, M.Y. Theoretical and experimental investigation of surface generation in swing process bonnet polishing of complex three-dimensional structured surfaces. Precis. Eng. 2017, 50, 361-371. [CrossRef]

21. Li, L.X.; Zheng, W.J.; Deng, L.G.; Wang, X.; Wang, X.K.; Zhang, B.Z.; Bai, Y.; Hu, H.X.; Zhang, X.J. Optimized dwell time algorithm in magnetorheological finishing. Int. J. Adv. Manuf. Technol. 2015, 81, 833-841. [CrossRef]

22. Han, Y.J.; Zhu, W.L.; Zhang, L.; Beaucamp, A. Region adaptive scheduling for time-dependent processes with optimal use of machine dynamics. Int. J. Mach. Tools Manuf. 2020, 156, 103589. [CrossRef]

23. Han, Y.J.; Duan, F.; Zhu, W.L.; Zhang, L.; Beaucamp, A. Analytical and stochastic modeling of surface topography in timedependent sub-aperture processing. Int. J. Mech. Sci. 2020, 175, 105575. [CrossRef]

24. Zheng, W.M.; Cao, T.N.; Zhang, X.Z. Applications of a novel general removal function model in the CCOS. Proc. Soc. Photo Opt. Instrum. Eng. 2000, 4231, 51-58.

25. Ghigo, M.; Canestrari, R.; Spigal, D.; Novi, A. Correction of high spatial frequency errors on optical surfaces by means of ion beam figuring. Proc. Soc. Photo Opt. Instrum. Eng. Conf. Ser. 2007, 6671, 667114. 